\title{
РУССКОЯЗЫЧНАЯ ВЕРСИЯ ОПРОСНИКА ЭТИЧЕСКИХ ПОЗИЦИЙ: РЕПЛИКАЦИЯ ВНУТРЕННЕЙ СТРУКТУРЫ
}

\begin{abstract}
А.А. ФЁДОРОВ
${ }^{a}$ Новосибирский государственный университет, 630090, Россия, Новосибирск, ул. Пирогова, д. 1

Резюме

В статье представлены результаты репликационного исследования, направленного на проверку стабильности двухуровневой ортогональной структуры русскоязычной версии опросника этических позиций Д.Р. Форсайта (Ethics Position Questionnaire). При помощи конфирматорного факторного анализа на выборке из 201 человека показано, что эта структура хорошо соответствует эмпирическим данным и характеризуется лучшей пригодностью по сравнению с оригинальной одноуровневой моделью Д.Р. Форсайта. Также в данном репликационном исследовании установлено, что EPQ имеет хорошую внутреннюю согласованность, конвергентную и дискриминантную валидность. Последняя оценивалась при помощи шкалы лжи краткой формы личностного опросника Айзенка, а не методики Марлоу-Крауна (как в оригинальном исследовании), что позволяет оценить не просто воспроизводимость, но и повторяемость результатов. Полученные в данном репликационном исследовании данные свидетельствуют о воспроизводимости двухуровневой ортогональной структуры EPQ.
\end{abstract}

Ключевые слова: этические позиции, идеализм, релятивизм, мораль, диспозиция, воспроизводимость, репликация.

В контексте «кризиса воспроизводимости» (Open Science Collaboration, 2012, 2015), который мало изменил исследовательскую практику отечественной психологии', все бо́льшую значимость начинают приобретать репликационные исследования. С нашей точки зрения, обязанность повторить исследование лежит, в первую очередь, на самом ученом, более того, оптимальная стратегия заключается в репликации до, а не после публикации полученных результатов. Цель данной работы заключается в репликации внутренней структуры русскоязычной версии опросника этических позиций Д.Р. Форсайта (Ethics Position Questionnaire, EPQ), отчет по валидизации которой был опубликован нами ранее (Фёдоров, Бадиев, 2018). В свете вышесказанного

\footnotetext{
${ }^{1}$ Более того, некоторые «постнеклассические» исследователи вообще предлагают исключить воспроизводимость из перечня важнейших критериев достоверности научных результатов (Вачков, Вачкова, 2016).
}

Работа выполнена при финансовой поддержке РФФИ, проект № 18-013-00715. 
следует признать, что последовательность репликации и публикации в данном случае не соответствует оптимальной стратегии, но сам анализ стабильности факторного решения по-прежнему представляет интерес и позволяет оценить достоверность полученных результатов.

\section{Методика}

Выборка. В исследовании принял участие 201 человек в возрасте от 17 до 53 лет $(\mathrm{M}=21.87, \mathrm{Me}=21, \mathrm{SD}=4.90), 80$ мужчин и 121 женщина.

Подвыборки по использованным методикам: опросник этических позиций EPQ - 201 чел., краткая форма личностного опросника Айзенка - 147 чел.

Данная выборка удовлетворяет средним по строгости эмпирическим требованиям, опирающимся либо на абсолютное число людей в выборке ( $>$ > 200), либо на соотношение числа людей к количеству переменных (N:p>10)².

\section{Инструменты}

1. Опросник этических позищий (EPQ) Д.Р. Форсайта создан для оценки идеализма и релятивизма и состоит из 20 пунктов, по 10 на каждую из шкал (Forsyth, 1980). В настоящем исследовалась валидизированная на российской выборке русскоязычная версия с 5-балльной шкалой ответов (Фёдоров, Бадиев, 2018).

2. Краткая форма личностного опросника Айзенка (Слободская и др., 2006) проводилась полностью, но использовались только показатели шкалы лжи для оценки дискриминантной валидности в контексте анализа смещения ответов, которое может быть вызвано социальной желательностью. Решение использовать шкалу лжи вместо задействованной в предыдущем исследовании методики Марлоу-Крауна обусловлено тем, что шкала лжи из опросников Айзенка является более чистым измерением традиционного компонента социальной желательности, который Д.Л. Полхус назвал «управлением впечатлением» (Paulhus, 1991). Кроме того, К. Драммонд рекомендует при репликации «преднамеренно увеличивать различия» между двумя исследованиями, что позволяет оценить не просто воспроизводимость, но повторяемость результата, которая и является золотым стандартом науки (Drummond, 2018).

\section{Результаты}

\section{Репликация факторной структуры $E P Q$}

Для анализа факторной структуры опросника EPQ проводился конфирматорный факторный анализ в статистическом пакете EQS 6.2 for Windows.

${ }^{2}$ Обзор критериев приведен в статье Р. Маккалума, К. Уидэмэна, С. Жанга и С. Хонга (MacCallum et al., 1999). В нем описываются как более мягкие (например, N > 100, N:p > 5), так и более жесткие эмпирические правила (например, N > 300). В целом выборку в 200 человек принято рассматривать как достаточную для большинства моделей, особенно относительно простых (Kline, 2015). 
Проверялись две теоретические модели. Модель 1 является двухфакторной и соответствует оригинальной методике Д.Р. Форсайта (Forsyth, 1980). Модель 2 была получена нами в предыдущем исследовании (Фёдоров, Бадиев, 2018), она состоит из пяти факторов первого порядка, которые образуют два ортогональных фактора второго порядка. Фактор второго порядка «идеализм» включает в себя два фактора первого порядка: «недопущение вреда» и «забота о благе». Фактор второго порядка «релятивизм» включает три фактора первого порядка: «относительность этических систем», «межличностный релятивизм» и «допустимость лжи». Апостериорная модификация моделей на основе индексов Лагранжа не использовалась.

Поскольку анализ, проведенный с помощью IBM SPSS Amos 19, показал, что распределение переменных отклоняется от многомерной нормальности (c.r. = 13.28), конфирматорный факторный анализ проводился с поправкой Саторры-Бентлера (Satorra, Bentler, 1994). Для оценки пригодности моделей использовались следующие показатели: 1) Satorra-Bentler (S-B) $\chi^{2}, p>0.05$; 2) $\mathrm{RMSEA}<0.05$ (при $90 \%$ CI от 0.000 до 0.049); 3) CFI > 0.95; 4) IFI > 0.95; 5) TLI > 0.95. Результаты проверки моделей представлены в таблице 1.

Как видно из приведенных данных, оригинальная двухфакторная модель продемонстрировала низкий уровень соответствия эмпирическим данным, в то время как разработанная в прошлом исследовании модель 2 показала отличную пригодность.

\section{Внутренняя согласованность шкал $E P Q$}

В данном репликационном исследовании в качестве меры внутренней согласованности рассматривался коэффициент $\alpha$ Кронбаха, вычислявшийся в программном пакете Statsoft STATISTICA 10. М и SD рассчитывались для средних баллов. Результаты анализа приведены в таблице 2.

Приведенные данные свидетельствуют о хорошей внутренней согласованности всех шкал и подшкал EPQ, кроме И2, коэффициент $\alpha$ для которой не достигает конвенционального референсного значения.

\section{Конвергентная и дискриминантная валидность шкал EPQ}

Конвергентная и дискриминантная валидность основных шкал EPQ оценивалась в рамках подхода Форнелла-Ланкера (Fornell, Larcker, 1981). В качестве

Таблица 1

Показатели соответствия моделей по итогам CFA

\begin{tabular}{|l|c|c|c|c|c|}
\hline \multicolumn{1}{|c|}{ Модель } & (S-B) $\boldsymbol{\chi}^{2}, \boldsymbol{p}$ & RMSEA (90\% CI) & CFI & IFI & TLI \\
\hline M.1 (Форсайт, 1980) & $\begin{array}{c}350.62, \\
p<0.001\end{array}$ & $\begin{array}{c}0.073 \\
(0.062,0.083)\end{array}$ & 0.838 & 0.840 & 0.817 \\
\hline $\begin{array}{l}\text { M.2 (Фёдоров, Бадиев, } \\
\text { 2018) }\end{array}$ & $\begin{array}{c}189.55, \\
p=0.075\end{array}$ & $\begin{array}{c}0.029 \\
(0.000,0.044)\end{array}$ & 0.976 & 0.977 & 0.972 \\
\hline
\end{tabular}


критериев конвергентной валидности использовались следующие значения: $\mathrm{AVE}>0.50, \mathrm{CR}>0.70, \mathrm{CR}>\mathrm{AVE}$. В качестве критериев дискриминантной валидности использовались следующие показатели: для любых двух факторов $\mathrm{A}$ и $\mathrm{B} A V \mathrm{E}_{\mathrm{A}}>\mathrm{r}_{\mathrm{AB}}{ }^{2}, \mathrm{AVEB}>\mathrm{r}_{\mathrm{AB}}^{2}$. Результаты анализа, свидетельствующие о хорошей конвергентной и дискриминантной валидности двухуровневой ортогональной модели $\mathrm{EPQ}$, приведены в таблице 3.

О независимости факторов идеализма и релятивизма свидетельствует также практически нулевая корреляционная связь между ними $(r=-0.08, p=0.242)$.

Также в рамках проверки дискриминантной валидности проверялась связь шкал и подшкал EPQ со шкалой лжи из краткой формы личностного опросника Айзенка (таблица 4). С учетом нарушение нарушения нормальности для шкалы лжи (Shapiro-Wilk W $=0.958, p<0.001$ ), использовался коэффициент ранговой корреляции Спирмена.

Ни одна из корреляционных связей не достигает уровня значимости, следовательно, содержание пунктов, входящих в шкалу, не смешано с социальной желательностью, в частности, с таким ее компонентом, как управление впечатлением.

Укажем, что различия по фактору пола не обнаружены ни для идеализма $(t=1.47, p=0.143)$, ни для релятивизма $(t=-0.47, p=0.638)$, что согласуется с результатами оригинального исследования.

Таблица 2

Коэффициенты $\alpha$ для шкал и подшкал EPQ

\begin{tabular}{|l|c|c|c|l|c|c|c|}
\hline \multicolumn{1}{|c|}{ Шкала } & $\boldsymbol{\alpha}$ Кронбаха & $\mathbf{M}$ & $\mathbf{S D}$ & \multicolumn{1}{c|}{ Шкала } & $\boldsymbol{\alpha}$ Кронбаха & $\mathbf{M}$ & SD \\
\hline Идеализм & 0.854 & 3.52 & 1.05 & Релятивизм & 0.805 & 3.45 & 1.04 \\
\hline И1 & 0.892 & 3.81 & 1.23 & Р1 & 0.743 & 3.54 & 1.14 \\
\hline И2 & 0.521 & 3.08 & 0.99 & Р2 & 0.680 & 3.11 & 1.25 \\
\hline & & & Р3 & 0.778 & 3.75 & 1.39 \\
\hline
\end{tabular}

Таблица 3

$\mathrm{AVE}, \mathrm{CR}, \mathbf{r}_{\mathrm{AB}}^{2}$ шкал EPQ

\begin{tabular}{|l|c|c|c|}
\hline \multicolumn{1}{|c|}{ Шкала } & AVE & $\mathbf{C R}$ & $\mathbf{r}_{\text {Aв }}{ }^{2}$ \\
\cline { 1 - 2 } Идеализм & 0.789 & 0.881 & 0 \\
\cline { 1 - 2 } Релятивизм & 0.567 & 0.790 & \\
\hline
\end{tabular}

Таблица 4

Корреляции между шкалами ЕРQ и шкалой лжи ЛОА-К (N=147)

\begin{tabular}{|c|c|c|c|c|c|c|c|}
\hline Шкала & И & P & И1 & И2 & P1 & P2 & P3 \\
\hline Шкала лжи & 0.112 & -0.156 & 0.141 & 0.006 & -0.134 & -0.130 & -0.129 \\
\hline
\end{tabular}




\section{Обсуждение результатов}

Проведенное репликационное исследование подтверждает вывод о том, что ортогональная двухуровневая модель опросника EPQ хорошо соответствует эмпирическим данным и характеризуется лучшей пригодностью по сравнению с оригинальной одноуровневой моделью Д.Р. Форсайта. Опросник вновь продемонстрировал хорошую внутреннюю согласованность, конвергентную и дискриминантную валидности и, следовательно, может использоваться в психологических исследованиях в качестве инструмента оценки общих этических позиций и их составляющих. И2 («забота о благе») является единственной подшкалой, которая с точки зрения конвенциональных норм характеризуется не очень высокой внутренней согласованностью. С нашей точки зрения, к данному случаю вполне применимо следующее высказывание Н. Шмитта: «Не существует сакрального уровня приемлемых или неприемлемых значений коэффициента альфа. В некоторых случаях инструменты с низкими (по конвенциональным стандартам) уровнями альфы могут быть весьма полезны» (Schmitt, 1996, р. 353). Кроме того, при оценке приемлемости/неприемлемости конкретного показателя альфы следует учесть, что он увеличивается вместе с ростом количества пунктов и что для шкал с тремя-четырьмя пунктами уровень 0.5 представляется вполне достаточным (при конвенциональной норме 0.6).

В завершение еще раз отметим, что, с нашей точки зрения, этические позиции представляют собой не гипотетические внутренние причины, а лишь вероятности поведения, сформировавшиеся в результате индивидуальной жизненной истории индивида. Этические позиции - не эксплананс, но экспланандум.

\section{Литература}

Вачков, И. В., Вачкова, С. Н. (2016). Воспроизводимость психологических экспериментов как проблема постнеклассической науки. Культурно-историческая психология, 12(1), 97-101.

Слободская, Е., Князев, Г. Г., Сафронова, М. В. (2006). Краткая форма личностного опросника Айзенка (ЛОА-К) и ее использование для оценки риска употребления психоактивных веществ. Психологический журнал, 27(3), 94-105.

Фёдоров, А. А., Бадиев, И. В. (2018). Валидизация русскоязычной версии опросника этических позиций. Психология. Журнал Высшей школь экономики, 15(3), 491-509. doi:10.17323/18138918-2018-3-491-509

Ссылки на зарубежные источники см. в разделе References после англоязычного блока.

Фёдоров Александр Александрович - заведующий кафедрой, кафедра клинической психологии, Институт медицины и психологии, Новосибирский государственный университет, кандидат психологических наук.

Сфера научных интересов: философия и история психологии, современный бихевиоризм, психология морального поведения.

Контакты: fedleks@yandex.ru 


\title{
The Russian-Language Version of the Ethics Position Questionnaire: Replication of the Internal Structure
}

\author{
A.A. Fedorov ${ }^{a}$ \\ ${ }^{a}$ Novosibirsk State University, 1 Pirogova Str., Novosibirsk, 630090, Russian Federation
}

\begin{abstract}
The aim of the present study was to replicate the two-level orthogonal structural model of the Russian version of D. R. Forsyth's Ethics Position Questionnaire (EPQ). The confirmatory factor analysis revealed that this model fits well the empirical data and has better fit indices than the original single-level model. The replication research also established that the EPQ has good internal consistency, convergent and discriminant validity. The latter was assessed using the Lie scale of the short form of the Eysenck Personality Questionnaire, and not the Marlowe-Crowne Social Desirability Scale (as in the original study). This allowed evaluating not only the reproducibility, but also the retestability of the results. The data obtained in this replication study indicate the reproducibility of the two-level orthogonal structure of the EPQ.
\end{abstract}

Keywords: ethics positions, idealism, relativism, morality, disposition, reproducibility, replication.

\section{References}

Drummond, C. (2018). Reproducible research: a minority opinion. Journal of Experimental and Theoretical Artificial Intelligence, 30(1), 1-11. doi:10.1080/0952813X.2017.1413140

Fedorov, A. A., \& Badiev, I. V. (2018). Validation of the Russian-language version of the Ethics Position Questionnaire. Psychology. Journal of the Higher School of Economics, 15(3), 491-509. doi:10.17323/1813-8918-2018-3-491-509 (in Russian)

Fornell, C., \& Larcker, D. F. (1981). Evaluating structural equation models with unobservable variables and measurement error.Journal of Marketing Research, 18(1), 39-50. doi:10.2307/3151312

Forsyth, D. R. (1980). A taxonomy of ethical ideologies. Journal of Personality and Social Psychology, 39(1), 175-184. doi:10.1037/0022-3514.39.1.175

Kline, R. B. (2015). Principles and practice of structural equation modeling (4th ed.). New York: The Guilford Press.

MacCallum, R. C., Widaman, K. F., Zhang, S., \& Hong, S. (1999). Sample size in factor analysis. Psychological Methods, 4(1), 84-99. doi:10.1037/1082-989X.4.1.84

Open Science Collaboration. (2012). An open, large-scale, collaborative effort to estimate the reproducibility of psychological science. Perspectives on Psychological Science, 7(6), 657-660. doi:10.1177/1745691612462588

Open Science Collaboration. (2015). Estimating the reproducibility of psychological science. Science, 349(6251), aac4716. doi:10.1126/science.aac4716 
Paulhus, D. L. (1991). Measurement and control of response bias. In Measures of Social Psychological Attitudes: Vol. 1. Measures of personality and social psychological attitudes (pp. 17-59). Academic Press. doi:10.1016/B978-0-12-590241-0.50006-X

Satorra, A., \& Bentler, P. M. (1994). Corrections to test statistics and standard errors in covariance structure analysis. In Latent variables analysis: Applications for developmental research (pp. 399-419). Thousand Oaks, CA: Sage.

Schmitt, N. (1996). Uses and abuses of coefficient alpha. Psychological Assessment, 8(4), 350-353. doi:10.1037/1040-3590.8.4.350

Slobodskaya, E. R., Knyazev, G. G., \& Safronova, M. V. (2006). Eysenck personality questionnaire brief form and its application for evaluation of adjustment and risk of psychoactive substances usage. Psikhologicheskii Zhurnal, 27(3), 94-105. (in Russian)

Vachkov, I. V., \& Vachkova, S. N. (2016). Reproducibility of psychological experiments as a problem of post-nonclassical science. Kul'turno-Istoricheskaya Psikhologiya [Cultural-Historical Psychology], 12(1), 97-101. doi:10.17759/chp.2016120110 (in Russian)

Alexandr A. Fedorov - Associate Professor, Chair of Clinical Psychology, Institute of Medicine and Psychology, Novosibirsk State University, PhD in Psychology.

Research Area: philosophy and theory of psychology, contemporary behaviorism, psychology of moral behavior.

E-mail: fedleks@yandex.ru 